\title{
BEHAVIOR OF MAXIMALLY DEFINED SOLUTIONS OF A NONLINEAR VOLTERRA EQUATION
}

\author{
TERRY L. HERDMAN
}

ABSTRACT. This paper is concerned with the behavior of solutions of an $n$-dimensional nonlinear Volterra integral equation

$$
x(t)=f(t)+\int_{0}^{t} g(t, s, x(s)) d s, \quad t>0 .
$$

In particular, sufficient conditions for a solution $x(t)$ on its maximal interval of existence $[0, T)$ to possess the property that $|x(t)| \rightarrow+\infty$ as $t \rightarrow T^{-}$are obtained. Thus these additional conditions give a positive answer to the problem posed by Miller [3, p. 145]. One can construct examples, satisfying the hypotheses given in [3], which provide a negative answer to this problem, see Artstein [1, Appendix A].

1. Introduction. In this paper we investigate the behavior of solutions of the $n$-dimensional nonlinear Volterra integral equation

$$
x(t)=f(t)+\int_{0}^{t} g(t, s, x(s)) d s,
$$

where $t$ belongs to a half open interval $[0, \eta)$, and the functions $x, f$ and $g$ have values in $R^{n}$, the real $n$-dimensional space. In particular, we shall investigate the behavior of $\lim |x(t)|$ as $t \rightarrow T^{-}$where $[0, T)$ is the maximal interval of existence for the solution $x(t)$. The function $f$ is assumed to be continuous on $R^{+}=[0, \infty)$, and we assume that the function $g$ satisfies the following hypotheses:

(H1) The function $g$ is defined for all $(t, s, x) \in R^{+} \times R^{+} \times R^{n}$, $g(t, s, x)=0$ whenever $s>t$ and $x \in R^{n}$; moreover, $g(t, s, x)$ is measurable in $s$ on $[0, t]$ for each $(t, x) \in R^{+} \times$ $R^{n}$, and $g(t, s, x)$ is continuous in $x$ for each fixed pair $(t, s) \in R^{+} \times R^{+}$.

(H2) For each real number $K>0$ and each bounded subset $B$ of $R^{n}$ there exists a measurable function $m$ such that

$$
|g(t, s, x)| \leqslant m(t, s) \quad(0 \leqslant s \leqslant t \leqslant K, x \in B)
$$

and

Received by the editors April 7, 1977.

AMS (MOS) subject classifications (1970). Primary 45D05, 45D99.

Key words and phrases. Nonlinear Volterra integral equation, maximal interval of existence, maximally defined solution. 


$$
\sup \left\{\int_{0}^{t} m(t, s) d s: 0 \leqslant t \leqslant K\right\}<\infty .
$$

(H3) For each compact subinterval $J$ of $R^{+}$, each bounded set $B$ in $R^{n}$ and each $t_{0}$ in $R^{+}$,

$$
\sup \left\{\int_{J}\left|g(t, s, \varphi(s))-g\left(t_{0}, s, \varphi(s)\right)\right| d s: \varphi \in C(J ; B)\right\} \rightarrow 0
$$

as $t \rightarrow t_{0}$.

We have from [3] the following information about equation (E), when (H1) - (H3) are satisfied.

(C1) There exist a number $\beta>0$ and a continuous function $x(t)$ such that $x(t)$ satisfies equation $(\mathrm{E})$ on $[0, \beta]$.

(C2) If $x(t)$ is a bounded continuous solution of (E) for $t \in[0, \alpha)$, where $\alpha<+\infty$, then $x(t)$ can be extended as a continuous solution of (E) to an interval $\left[0, \alpha_{0}\right]$ where $\alpha_{0}>\alpha$.

(C3) Each continuous solution of (E) can be extended to the right to obtain a maximally defined solution (i.e. a continuous solution $x(t)$ of $(\mathrm{E})$ defined on an interval $[0, \alpha)$ where either $\alpha=+\infty$ or $\lim \sup |x(t)|=\infty$ as $t \rightarrow \alpha^{-}$. The interval $[0, \alpha)$ is called the maximal interval of existence for the solution $x(t)$.)

In [1], Artstein obtained these results under somewhat weaker but similar assumptions on the function $g$ in equation (E). Herdman [2] obtained these results under different hypotheses.

From (C3) we see that if $x(t)$ is a continuous solution of $(\mathrm{E})$ on $[0, \eta)$ which cannot be extended, then either $\eta=+\infty$ or lim sup $|x(t)|=\infty$ as $t \rightarrow \eta^{-}$. Artstein [1, Appendix A] constructed an example $x:[0,1) \rightarrow R$ where lim $\sup |x(t)|=+\infty$ as $t \rightarrow 1^{-}$but $\lim |x(t)| \neq \infty$ as $t \rightarrow 1^{-}$. This example satisfied hypothesis (H1)-(H3) of this paper and (H7) of [3]. Thus, it is an answer to the open problem posed by Miller [3, Problem 15, p. 145]. This example gave a negative answer for the open problem.

The purpose of the present paper is to present sufficient conditions for a continuous solution $x(t)$ of $(\mathrm{E})$ on its maximal interval of existence $[0, T)$ to possess the property that $|x(t)| \rightarrow \infty$ as $t \rightarrow T^{-}$. In stating additional hypothesis for the function $g$, we need the following vector notation. For a general $n$-vector $M=\left(M_{\alpha}\right)$ the symbol $M \cdot \geqslant \cdot 0,\{M \cdot>\cdot 0\}$, signifies that the elements of $M$ are real, and $M_{\alpha} \geqslant 0,\left\{M_{\alpha}>0\right\}$, for $\alpha=1, \ldots, n$. The notation $M \cdot \geqslant \cdot N$ is used to signify $M-N \cdot \geqslant \cdot 0$, with similar meaning for $M \cdot>\cdot N, M \cdot<\cdot N$ and $M \cdot \leqslant \cdot N$. The symbol 0 is used indiscriminately for the zero vector of any dimension.

We now list two additional hypotheses for the function $g$ in equation (E). 
(H4) For each $\alpha>0$ there exists a constant $n$-vector $K_{\alpha} \cdot \geqslant \cdot 0$ such that $g(s, s, x) \cdot \geqslant-K_{\alpha}$ for $(s, x) \in[0, \alpha] \times R^{n}$.

(H5) For each $\alpha>0$ there exists a constant $n$-vector $L_{\alpha} \cdot \geqslant \cdot 0$ such that

$$
g(t, s, x)-g(\tau, s, x) \cdot \geqslant-L_{\alpha}[t-\tau]
$$

for $0 \leqslant s \leqslant \tau \leqslant t \leqslant \alpha$ and $x \in R^{n}$.

Our main result is the following theorem.

THEOREM 1. Let the function $g$ of (E) satisfy (H4) and (H5). If $x(t)$ is a maximally defined solution of equation $(\mathrm{E})$, with maximal interval of existence $[0, T)$ where $T<\infty$ then $\lim |x(t)|=+\infty$, as $t \rightarrow T^{-}$.

By way of comments, we note that the existence of a maximally defined solution is part of the hypothesis. Applying the results of [3], mentioned earlier in this paper, we see that equation (E) has a local solution and each solution can be extended to obtain a maximally defined solution. An example where the maximal interval of existence is of the form $[0, T), T<+\infty$ will be given in $\S 4$.

To conclude this section we state a lemma which will be used in the proof of Theorem 1.

LEMMA 1. Let $y:[0, T) \rightarrow R^{n}$, where $0<T<+\infty$, be a continuous function satisfying

(1.1) $\lim _{t \rightarrow T^{-}}|y(t)| \neq+\infty$,

(1.2) there exists a constant $n$-vector $B \cdot \geqslant \cdot 0$ such that $y(t)-y(\tau) \cdot \geqslant$ $-B(t-\tau)$ for $0 \leqslant \tau \leqslant t<T$.

Then the limit of $y(t)$ as $t \rightarrow T^{-}$exists.

2. Proof of Lemma 1. Attention will be focused upon the case where $y$ is a real valued function. In particular, the inequality, $\cdot \geqslant \cdot$, in (1.2) will now be the usual, $\geqslant$, inequality for real values. The proof for the $n$-dimensional vector equation will follow immediately.

The hypothesis readily implies that there exists a sequence $\left\{t_{n}\right\}$, where $0<t_{n}<t_{n+1}<T$ for $n=1,2, \ldots$, and a point $y_{0}$ such that $\left\{t_{n}\right\}$ converges to $T$ and $y\left(t_{n}\right)$ converges to $y_{0}$ as $n$ approaches $+\infty$.

Let $\varepsilon>0$ be given, and let $\left\{\hat{t_{j}}\right\}$ be an arbitrary increasing sequence of values in $[0, T)$ such that $\hat{t}_{j} \rightarrow T^{-}$as $j \rightarrow+\infty$. We choose $M_{1}$, sufficiently large, such that $\left|y\left(t_{n}\right)-y_{0}\right|<\varepsilon / 3$ and $0<T-t_{n}<\varepsilon / 3(B+1)$ whenever $n>M_{1}$. Now pick $M_{2}$ such that $t_{M_{1}} \leqslant \hat{t_{j}}<T$ for all $j \geqslant M_{2}$. It is to be noted that for each $j \geqslant M_{2}$ there is a $n_{j}>M_{1}$ such that $t_{M_{1}} \leqslant \hat{t_{j}}<t_{n_{j}}$, where $t_{n_{j}} \in\left\{t_{n}\right\}$.

In view of inequality (1.2), we have that

$$
y\left(t_{n_{j}}\right)-y\left(\hat{t_{j}}\right) \geqslant-B\left(t_{n_{j}}-\hat{t}_{j}\right)>-B \varepsilon / 3(B+1)>-\varepsilon / 3
$$

for all $j \geq M_{2}$. Thus it follows that 


$$
y\left(\hat{t}_{j}\right)<y\left(t_{m_{j}}\right)+\varepsilon / 3<y_{0}+\varepsilon
$$

for $j \geqslant M_{2}$. On the other hand,we have that

$$
y\left(\hat{t}_{j}\right)-y\left(t_{M_{1}}\right) \geqslant-B\left(\hat{t}_{j}-t_{M_{1}}\right) \geqslant-B \varepsilon / 3(B+1) \geqslant-\varepsilon / 3
$$

for $j \geqslant M_{2}$. Consequently, it follows that

$$
y\left(\hat{t}_{j}\right) \geqslant y\left(t_{M_{1}}\right)-\varepsilon / 3>y_{0}-\varepsilon
$$

for $j \geqslant M_{2}$. In particular, inequalities (2.1) and (2.2) imply that the sequence $\left\{y\left(\hat{t}_{j}\right)\right\}$ converges to $y_{0}$ as $j \rightarrow+\infty$. In view of the arbitrariness of the increasing sequence $\left\{\hat{t}_{j}\right\}$ converging to $T$, we have that the limit of $y(t)$, as $t \rightarrow T^{-}$, equals $y_{0} \in R$.

\section{Proof of Theorem 1. Define $y(t)$ by}

$$
y(t)=x(t)-f(t) \text { for } t \in[0, T) .
$$

From (3.1) and the hypothesis it follows that $y \in C\left([0, T) ; R^{n}\right)$.

To prove Theorem 1 we assume that the conclusion does not hold, that is, $\lim |x(t)| \neq+\infty$ as $t \rightarrow T^{-}$. It is evident from (3.1) and the above assumption that $\lim |y(t)| \neq+\infty$ as $t \rightarrow T^{-}$.

We claim that

$$
\lim x(t) \text { as } t \rightarrow T^{-} \text {exists. }
$$

As a first step in establishing (3.2) we assert that the function $y$ defined by (3.1) satisfies the hypothesis of Lemma 1.

Let $t$ and $\tau$ be elements of $[0, T)$ satisfying $0 \leqslant \tau \leqslant t<T$. In view of (3.1) (H4) and (H5) one has

$$
\begin{aligned}
y(t)-y(\tau)= & x(t)-f(t)-x(\tau)+f(\tau) \\
= & \int_{0}^{t} g(t, s, x(s)) d s-\int_{0}^{\tau} g(\tau, s, x(s)) d s \\
= & \int_{0}^{\tau}\{g(t, s, x(s))-g(\tau, s, x(s))\} d s \\
& +\int_{\tau}^{t}\{g(t, s, x(s))-g(s, s, x(s))\}+\int_{\tau}^{t} g(s, s, x(s)) d s \\
& \geqslant \cdot \int_{0}^{\tau}-L_{T}(t-\tau) d s+\int_{\tau}^{t}-L_{T}(t-s) d s+\int_{\tau}^{t}-K_{T} d s \\
& \geqslant \cdot-L_{T}(\tau)(t-\tau)-L_{T} \frac{(t-\tau)^{2}}{2}-K_{T}(t-\tau) \\
& \geqslant \cdot(t-\tau)\left[-L_{T}(T)-L_{T}(T)-K_{T}\right] .
\end{aligned}
$$

Thus it follows that

$$
y(t)-y(\tau) \cdot \geqslant-B(t-\tau)
$$

for $0 \leqslant \tau \leqslant t<T$, where $B=2 T L_{T}+K_{T} \cdot \geqslant \cdot 0$. As a consequence of Lemma 1, it follows that the limit of $|y(t)|$ as $t \rightarrow T^{-}$exists. Since $f$ is a 
continuous function on $[0, T)$ we have that limit of $x(t)$ as $t \rightarrow T^{-}$exists. Thus (3.2) is established.

It is an elementary consequence of (3.2) that $x(t)$ is bounded on $[0, T)$. In view of the result (C2) we have that there is a $T_{0}>T$ such that $x(t)$ can be extended, continuously, as a solution of $(E)$ on the interval $\left[0, T_{0}\right]$. This violates the interval $[0, T)$ being the maximal interval of existence for the solution $x(t)$. From this contradiction we finally deduce that $|x(t)| \rightarrow+\infty$ as $t \rightarrow T^{-}$.

To conclude this section we note that the lower Lipschitz condition of (H5) and the lower bound of (H4) can be replaced by a corresponding upper Lipschitz condition and a corresponding upper bound. In particular, the conclusion of Theorem 1 holds when (H4) and (H5) are replaced by

For each $\alpha>0$ there exist constants $k_{\alpha i}>0, l_{\alpha i} \geqslant 0$, $i \in N=\{1,2,3, \ldots, n\}$ and sets $I_{1}, I_{2}$ such that

(i) $I_{1} \cup I_{2}=N, I_{1} \cap I_{2}=\varnothing$;

(ii) $g_{i}(s, s, x) \geqslant-k_{\alpha i}$ for $(s, x) \in[0, \alpha] \times R^{n}$ and $i \in I_{1}$;

(iii) $g_{i}(s, s, x) \leqslant k_{\alpha i}$ for $(s, x) \in[0, \alpha] \times R^{n}$ and $i \in I_{2}$;

(iv) $g_{i}(t, s, x)-g_{i}(\tau, s, x) \geqslant-l_{\alpha i}[t-\tau]$ for $0<s<\tau<$ $t<\alpha, x \in R^{n}, i \in I_{1}$

(v) $g_{i}(t, s, x)-g_{i}(\tau, s, x) \leqslant l_{\alpha i}[t-\tau]$ for $0 \leqslant s \leqslant \tau \leqslant t$ $\leqslant \alpha, x \in R^{n}, i \in I_{2}$.

4. Remarks. As an immediate consequence of Theorem 1 we have the following corollary. Again, we are assuming (H1), (H2) and (H3).

COROLlary 1. Let

$$
\begin{gathered}
f^{\prime} \in C\left([0, T], R^{n}\right), \quad\left({ }^{\prime}=d / d t\right) \\
g_{t}(t, s, x) \cdot \geqslant-M_{T}, \quad \text { where } M_{T} \cdot>\cdot 0,0 \leqslant s \leqslant t<T, x \in R^{n} \\
g(t, t, x) \cdot \geqslant \cdot-K, \quad \text { where } K \cdot \geqslant \cdot 0 \\
\frac{d}{d t}\left(\int_{0}^{t} g(t, s, x(s)) d s\right)=g(t, t, x(t))+\int_{0}^{t} g_{t}(t, s, x(s)) d s
\end{gathered}
$$

for $t \in[0, T)$ and let $x(t)$ be a maximally defined solution of (E) on $[0, T)$. Then $|x(t)| \rightarrow+\infty$ as $t \rightarrow T^{-}$.

To realize this Corollary one can take the derivative of $x(t)$ to obtain

$$
x^{\prime}(t)=f^{\prime}(t)+g(t, t, x(t))+\int_{0}^{t} g_{t}(t, s, x(s)) d s, \quad t \in[0, T) .
$$

Applying conditions (4.1), (4.2) and (4.3) it follows that

$$
x^{\prime}(t) \cdot \geqslant-B-K-M_{T} T \text { for } t \in[0, T)
$$

where $B$ is the constant $n$ vector $\left(B_{i}\right), B_{i}=\sup \left\{\left|f^{\prime}(t)\right|: t \in[0, T]\right\}$.

Inequality (4.5) together with reasoning similar to that given in the proof of Theorem 1 completes the proof.

Consider the equation 


$$
x(t)=x_{0}+K \int_{0}^{t}(t-s) x(s)^{1+a} d s
$$

where $x_{0}, K$ and $a$ are positive constants. Equation (4.6) can be solved and the solution $x(t)$ is defined implicitly by

$$
\left(\frac{2 K}{2+a}\right)^{1 / 2} t=\frac{1}{\sqrt{x_{0}^{a}}} \int_{1}^{x(t) / x_{0}}\left(s^{2+a}-1\right)^{-1 / 2} d s .
$$

As seen $n$ Miller [3, pp. 46-48], solutions of equation (4.6) have finite escape times. The maximal interval of existence for a solution defined by (4.7) is seen to be $[0, T)$ where $T$ satisfies

$$
\left(\frac{2 K}{2+a}\right)^{1 / 2} T=x_{0}^{-a / 2} \int_{1}^{\infty}\left(s^{2+a}-1\right)^{-1 / 2} d s
$$

We note that the kernel function

$$
g(t, s, x)=K(t-s) x^{1+a}
$$

for equation (4.6) satisfies hypotheses (H4) and (H5) with $K_{T}=0$ and $L_{T}=0$. Therefore, Theorem 1 implies that $|x(t)| \rightarrow \infty$ as $t \rightarrow T^{-}$.

\section{REFERENCES}

1. Z. Artstein, Continuous dependence of solutions of Volterra integral equations, SIAM J. Math. Anal. 6 (1975), 446-456.

2. T. L. Herdman, Existence and contimuation properties of solutions of a nonlinear Volterra equation, Dynamical Systems, An International Symposium, Vol. 2, Academic Press, New York, 1976, pp. 307-310.

3. R. K. Miller, Nonlinear Volterra integral equations, W. A. Benjamin, Inc., Menlo Park, Calif., 1971.

Department of Mathematics, Virgina Polytechnic Institute and State University, BLACKSBURG, Virgini 24061 